\title{
Nursing interventions to minimize cetuximab-induced dermatologic toxicity
}

Helle M. Larsen, Mette Thode, Karin B. Dieperink, Jon Bjerregaard, Per Pfeiffer

Department of Oncology, Odense University Hospital, Denmark

Received: August 25, 2016

Accepted: December 28, 2016 Online Published: February 13, 2017

DOI: $10.5430 /$ cns.v5n $1 \mathrm{p} 45$

URL: http://dx.doi.org/10.5430/cns.v5n1p45

\begin{abstract}
Objective: This study investigated early nursing interventions with the purpose to minimize cetuximab-induced acneiform eruption. The most important side-effect of cetuximab is dermatologic toxicity, up to $90 \%$. Dose-reduction or interruption of cetuximab reduces severity of dermatologic toxicity, probably at the cost of reduced efficacy of the cancer therapy. Thus, prevention or effective supportive care during treatment is important. The study evaluated if patient education could ensure compliance of cancer treatment and effect of oral tetracycline on acneiform eruption, with the purpose to minimize severity of dermatologic toxicity and reduce the use of tetracycline.

Methods: The design was a single group prospective interventional study. Gastro-intestinal (GI) cancer patients treated with cetuximab between April 2009 and June 2011 were educated to start treatment with tetracycline $500 \mathrm{mg}$ twice daily when acneiform eruption occurred. Patient's dermatologic toxicity were graded (by CTCAE) and registered by nurses.

Results: Sixty-three patients were evaluable. Patients started tetracycline when acneiform eruption occurred. It reduced severity but not incidence of acneiform eruption, $10 \%$ of the patients never developed acneiform eruption and therefore never received tetracycline.

Conclusions: Patient-education by a trained oncology nurse in the handling of cetuximab induced acneiform eruption is manageable and effective and ensures a high number of patients carrying through treatment with a full dose of cetuximab.
\end{abstract}

Key Words: Nursing interventions, Skintoxicity, Cetuximab, Acneiform eruption, Rash, Dermatologic toxicity, Patient education, Tetracycline, Guideline

\section{INTRODUCTION}

Gastro-intestinal (GI) cancer is one of the most common causes of cancer-related death in the Western countries. ${ }^{[1]}$ Since publication of the pivotal BOND study, ${ }^{[2]}$ cetuximab has been part of the therapeutic arsenal in patients with metastatic colorectal cancer (mCRC). Cetuximab was first introduced in chemo-resistant $\mathrm{mCRC}$, but was later approved in all lines ${ }^{[3]}$ in combination with chemotherapy. Recently it was shown that cetuximab should only be administered in patients with rat sarcoma (RAS) wild type tumours. ${ }^{[4]}$

Cetuximab is associated with dermatological toxicity that affects up till $90 \%$ of the patients, which may affect compliance with the cancer treatment. ${ }^{[5]}$ If severe skin toxicity is prevented or limited it might minimize the need for dosereduction, dose-delay or discontinuation and it is natural to assume that an increased dose-intensity will optimize efficacy of cetuximab.

Dermatologic toxicity includes acneiform eruption, hyper-

\footnotetext{
* Correspondence: Helle M. Larsen; Email: helle.mona.larsen@rsyd.dk; Address: Department of Oncology, Odense University Hospital, Southern Boulevard 29, DK-5000 Odense C, Denmark.
} 
pigmentation, xerotic skin, pruritus, skin fissures and nail changes. Severe dermatologic toxicity is most often seen during the first two months of therapy. Dermatologic toxicity is identified in the literature with different terms e.g. rash, acne, acne-like skin rash, acneiform eruption. In this paper we use the term acneiform eruption expressed by Baas and colleagues. ${ }^{[5]}$ The side effects of cetuximab differ from chemotherapy induced toxicity and therefore needs special attention. In general, it is assumed that severe dermatologic toxicity reduces patients' quality of life.

Since the first randomized study was presented, ${ }^{[6]}$ we have offered oral tetracycline as part of our treatment strategy for acneiform eruption. Scope et al. showed in a randomized controlled trial $(n=48)$ that prophylactic minocycline reduced the severity but not the incidence of acneiform eruption. $^{[7]}$

In the BOND study, ${ }^{[2]}$ almost $20 \%$ of patients treated with cetuximab never developed acneiform eruption. Using a preemptive broad-spectrum antibiotic like tetracycline may thus increase unnecessary consumption and due to our concern about patients' risk of developing antibiotic resistance, we decided to examine whether it was possible to avoid or minimize duration of tetracycline treatment.

To the best of our knowledge, no study has examined nursing interventions in the management of cetuximab induced dermatologic toxicity. Previously, our treatment strategy for dermatologic toxicity was rather coincidental and evaluation and treatment of acneiform eruption was often not initiated until moderate or even severe acneiform eruption developed.

Therefore, we performed a pilot study in all patients starting cetuximab biweekly between April and June 2009 ( $n=26)$. Patients had GI cancer, and were unselected regarding age and gender. ${ }^{[8-10]}$ In connection with the first out-patient visit for cetuximab therapy, patients were educated by the treating nurse on toxicity of cetuximab with special attention on skin toxicity including how and when to start tetracycline. In the pilot study, patients self-administered tetracycline at a dosage of $333 \mathrm{mg}-1,000 \mathrm{mg}$ daily for an indefinite period of time. Results from the pilot study showed high compliance with tetracycline and a reduced severity of acneiform eruption. Therefore we decided to continue with a larger study, with the objective to confirm results from the pilot study.

\section{METHOD}

The design was a single group prospective interventional study executed in a Danish University Hospital at an oncology center. The study consisted of nursing management and patient-education related to acneiform eruption caused by cetuximab.

\subsection{The nursing role}

Given that nurses are the interface between the patient and the physician, we investigated the effect of "early onset" of treatment with tetracycline, including systematic patient education by oncology nurses and investigated if this strategy could minimize the severity of acneiform eruption. We wanted to focus on patients' resources and capacity to use their resources available by transferring the onset of treatment from health professional to patient. Thus, the nursing intervention was inspired by Antonovsky and the philosophy of salutogenesis, ${ }^{[11]}$ and the concepts of health promotion. ${ }^{[12]}$

\subsection{Patients}

All patients with GI cancer treated with cetuximab between April 2009 and May 2011 were informed and included by the study nurses. Patients were unselected regarding age and gender. Medical information was obtained by review of all individual medical records and included diagnosis, gender and age.

\subsection{Trial interventions}

Patients were systematically educated by trained oncology nurses after the guideline used in the pilot study, consisting of written information about the study and procedures. Furthermore, patients were by the nurses instructed in precautions to tetracycline, e.g. dyspeptic genes, skin photosensitivity, and interaction with dairy products. Dairy products might inactivate the effect of tetracycline and thus patients were instructed to avoid dairy products 3 hours before and after intake of tetracycline. Patients were given 30 pieces of tetracycline $500 \mathrm{mg}$, to ensure immediately start of treatment in the patient's home, at the earliest onset of acneiform eruption. Patients were also instructed to consult one of three named nurses by telephone when acneiform eruption appeared. Regardless the grade of acneiform eruption, treatment with tetracycline $500 \mathrm{mg}$ twice a day for 8 weeks was started.

\subsection{Method of evaluation}

In the patient record the nurse documented the date of start and the planned termination date after 8 weeks. In collaboration with dermatologists we prepared a treatment strategy with recommendations for further treatment in case of severe skin toxicity e.g. topical medications, cream, lotion, and other medications was registered. Recommendations could be used after the nurse or the physician assessment. All patients were advised on prophylactic skincare with lotion or cream without alcohol and perfume from the beginning of treatment. At each visit in the out-clinic for cetuximab treatment, the patient's side effects were registered by the nurse following the data collection tool CTCAE version 3.0. The American National Cancer Institute (NCI) has developed 
standardized definitions for adverse events - CTCAE (Common Criteria for adverse events) to describe the severity of organ toxicity for patients receiving cancer therapy. ${ }^{[13]}$

\subsection{Ethical considerations}

Data was collected with the permission of the Danish Data Protection agency. All patients accepted to participate in the study, and could withdraw their consent at any time.

\subsection{Statistics}

Socio-demographic and clinical characteristics of all patients were described by medians and ranges. Survival was estimated using the Kaplan-Meier method, from time of first infusion till death or last follow-up date. Data on rash severity was performed as a landmark analysis only including patients receiving at least 2 series of cetuximab.

\section{Results}

\subsection{Patient population}

Between April 2009 and June 2011, 75 patients started biweekly cetuximab (with chemotherapy) and were enrolled in the present study. Thirty-eight patients had $\mathrm{mCRC}^{[8,10]}$ and 37 had esophageal or gastric cancer. ${ }^{[9]}$ Sixty-three of these patients received at least 1 month of therapy with cetuximab, and were evaluable for intervention analysis. The median age was 63 years (range 41-77). Thirty-five percent were female.

\subsection{Rash}

The median reported time to rash development was 10 days (95\% CI 8-14). Six patients (10\%) did not develop any rash during the study period. Only one patient developed grade 3 skin rash (2\%). The remainder (88\%) developed grade 1-2 rash. Different grades of acneiform eruption are shown in Figure 1.

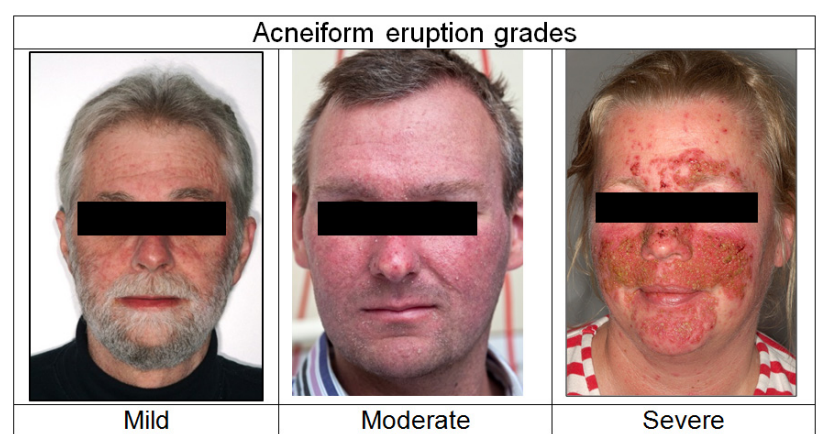

Figure 1. Grades of acneiform eruption according to CTCAE 3.0

Five patients $(8 \%)$ had dose reduction of cetuximab due to dermatologic toxicity. The reported severity of rash did not differ significantly between gender and age. The severity of rash per number of cycles given is illustrated in Figure 2.

\subsection{Compliance to intervention \& therapy}

Thirty-nine $(62 \%)$ of the patients contacted the department as instructed before initiation of tetracycline. The median time treated with tetracycline was 56 days (range 5-119). Twelve patients (19\%) needed to resume tetracycline after the pre-specified 8 week duration. The median time from planned stop to restart in these patients was 49 days (range 26-100 days).

Patients received a median of 8 (range 3-29) cycles of cetuximab. Overall seven patients were dose reduced, five due to rash and two due to hypomagnesaemia.

\subsection{Survival}

The median survival for evaluable patients was 13.3 months (CI 9.5-17.7). Patients with CRC survived longer (17.6 months [CI 13-NR]) than patients with esophageal/gastric cancer (7.7 months [CI 4.7-11.1]).

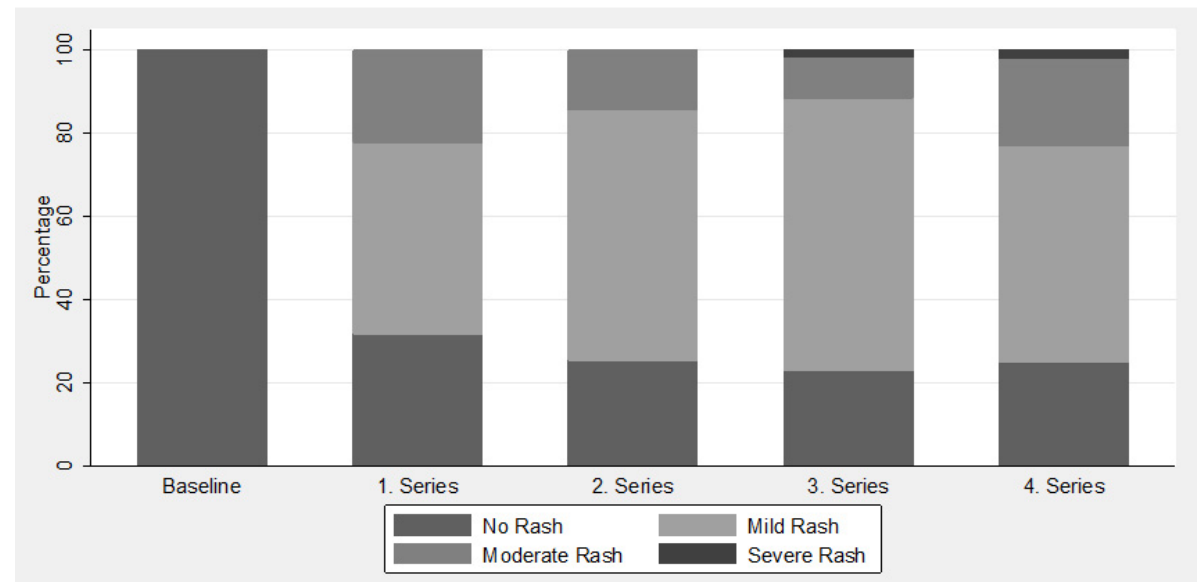

Figure 2. The severity of rash per number of cycles in $n=63$ gastro-intestinal cancer patients treated with cetuximab in Denmark 


\section{DiscuSSION}

\subsection{Reflections}

During this single center prospective study, we systematically organized the treatment with tetracycline, so only patients with acneiform eruption started the treatment at the right moment and for a defined period of time. One aim of our intervention was to strengthen the patients to be able to manage their own treatment of acneiform eruption. This aim succeeded and is in line with Ouwerkerk et al., ${ }^{[14]}$ who claimed that oncology nurses can help patients maintain their quality of life, promoted treatment adherence by empowering patients to be more involved in their treatment and in the management of treatment-related toxicities.

In the first prophylactic studies, tetracycline was continued as long as cetuximab was administered. In accordance with the strategy used by Scope et al., ${ }^{[7]}$ we decided to limit the duration of tetracycline therapy to 8 weeks at a dose of $500 \mathrm{mg}$ twice daily.

A lower dose of tetracycline might have the same effect, but this was not part of the present study and needs to be investigated in further studies.

In the randomized study by Scope et al., ${ }^{[7]}$ there was no difference in severity of skin toxicity after eight weeks of treatment with cetuximab. These results convinced us not to continue with tetracycline beyond 8 weeks. However we chose to continue evaluation of toxicity and evaluate a possible flair of symptoms which happened in $20 \%$ of patients. Those patients were urged to resume therapy with tetracycline $500 \mathrm{mg}$ twice daily for another four weeks, followed by a new planned treatment break and re-evaluation.

\subsection{Strength}

A strength of our study was the prospective design, and the instant start of tetracycline probably contributed to the low grade of skin toxicity and helped to maintain full dose intensity without unnecessary delay of cetuximab treatment for most of the patients. Moreover the need for additional supportive treatment e.g lotion and topical steroids was reduced

One important aim of our study, was to prevent administration of unnecessary tetracycline, which succeeded in $10 \%$ of the patients who never developed skin toxicity. We found that patients who developed acneiform eruption had their first rash at day 10. This is in contrast to Scope et al., who found the initial rash between days 14 and $28 .{ }^{[7]}$ In their review Ouwerkerk et al. found that $80 \%$ of the patients developed acneiform eruption within the first two weeks. Therefore it is essential for patients to start tetracycline at home, especially in patients who are being treated with cetuximab every second week. ${ }^{[14]}$ In relation to development of resistance, we might question continuation of prophylactic antibiotic for an indefinite time period. This must be weighed against treatment with cetuximab in the optimal dose intensity to secure optimal efficacy. As nurses and oncologist we must secure optimal treatment, but also avoid unnecessary use of antibiotics. In our study we found that $10 \%$ of patients never started treatment with tetracycline and additionally our systematic approach secured, that all patients terminated prophylactic tetracycline after a planned treatment period.

The major difference between our study and Scope et al. ${ }^{[7]}$ was that our patients evaluated their own acneiform eruption and called a named nurse immediately at the time of the first eruption. This secured an immediate start of tetracycline and registration of start date.

Around 10\%-15\% of cetuximab treated patients will develop grade 3 or 4 skin toxicity without use of prophylactic tetracycline. In our study only one of 63 patients $(2 \%)$ developed grade 3 toxicity. In clinical practice (grade 3 or higher) skin toxicity is a reason for dose reduction or interruption. It is likely that this might have a negative influence on treatment outcome. Only 5 patients (8\%) in our study had dose reduction of cetuximab and no patient discontinued cetuximab due to skin toxicity. This is much lower than expected from larger prospective studies.

To have cancer causes loss of control, ${ }^{[15]}$ and with this empowering nursing intervention, we believe some of the control was returned to the patients as the patients expressed they were well informed and comfortable by starting tetracycline at home. Furthermore, our guideline provided consensus in the information to the patients.

\subsection{Limitations}

Although our study was prospective, one important limitation was the lack of a control group to understand if our results were related to our nursing intervention. We did not register the need for consultation by oncologist before and during the study, but the nurses are experiencing a decreased demand for physician consultation. Physicians in our department recall that they rarely see patients with severe skin toxicity anymore and they are rarely involved in the handling of skin toxicity. Also, patients' behaviors might be changed because of the relation to a named nurse. They possibly felt more responsible because of the relation.

The nurses involved were especially trained to evaluate skin toxicity with CTCAE (version 3.0). Scope et al. used photo documentation and dermatologist to evaluate the severity of skin toxicity. ${ }^{[7]}$ In contrast our patients were evaluated by experienced oncology nurses and only patients with the most severe eruption were referred to a dermatologist. This new 
approach demands systematic education of oncology nurses, which may transfer costs between different sections of the health system.

\section{Conclusions}

This prospective nursing intervention study found that patient-education by a trained oncology nurse in the handling of cetuximab induced acneiform eruption is manageable and effective. The systematic approach ensured a high number of cancer patients carrying through cancer treatment with a full dose of cetuximab, and spared $10 \%$ of the cancer patients from unnecessary prophylactic tetracycline, and secured, that all patients terminated after a planned treatment period.
Our guideline was manageable for both patients and nurses. It resulted in uniformity and experience of security among physician and nurses, and made the process for patients being treated with cetuximab more efficient.

\section{Clinical perspectives}

The guideline we used for early patient education by nurses and immediately onset of tetracycline when occurrence of acneiform eruption may be used in other clinical settings. But it requires trained nurses who will assume the responsibility of handling the dermatologic toxicity.

\section{CONFLICTS OF INTEREST Disclosure}

The authors declare they have no conflict of interest.

\section{REFERENCES}

[1] Ferlay J, Soerjomataram I, Dikshit R, et al. Cancer incidence and mortality worldwide: sources, methods and major patterns in GLOBOCAN 2012. International Journal of Cancer. 2015; 136(5): E359E386. PMid: 25220842. https ://doi.org/10.1002/ijc. 2921 0

[2] Cunningham D, Humblet Y, Siena S, et al. Cetuximab monotherapy and cetuximab plus irinotecan in irinotecan-refractory metastatic colorectal cancer. New England Journal of Medicine. 1936; 351(351): $337-45$.

[3] Schmoll HJ, Cutsem EV, Stein A, et al. ESMO Consensus Guidelines for management of patients with colon and rectal cancer. A personalized approach to clinical decision, making. Annals of Oncology. 2012; 23(10): 2479-2516. PMid: 23012255. https: //doi.org/10.1093/annonc/mds236

[4] Cutsem EV, Lenz HJ, Köhne CH, et al. Fluorouracil, Leucovorin, and Irinotecan Plus Cetuximab Treatment and RAS Mutations in Colorectal Cancer. Journal of Clinical Oncology Official Journal of the American Society of Clinical Oncology. 2015; 33(7): 692-700. PMid: 25605843. https://doi.org/10.1200/JC0.2014.59.4812

[5] Baas JM, Krens LL, Guchelaar HJ, et al. Recommendations on management of EGFR inhibitor-induced skin toxicity: a systematic review. Cancer Treatment Reviews. 2012; 38(5): 505. PMid: 22100458. https://doi.org/10.1016/j.ctrv.2011.09.004

[6] Jatoi A, Rowland K, Sloan JA, et al. Tetracycline to prevent epidermal growth factor receptor inhibitor-induced skin rashes: results of a placebo-controlled trial from the North Central Cancer Treatment Group (N03CB. Cancer. 2008; 113(4): 847-853. PMid: 18543329. https://doi.org/10.1002/cncr. 23621

[7] Scope A, Agero ALC, Dusza SW, et al. Randomized Double-Blind Trial of Prophylactic Oral Minocycline and Topical Tazarotene for Cetuximab-Associated Acne-Like Eruption. Journal of Clini- cal Oncology. 2008; 25(34): 5390-5396. PMid: 18048820. https : //doi.org/10.1200/JC0.2007.12.6987

[8] Pfeiffer P, Nielsen D, Bjerregaard J, et al. Biweekly cetuximab and irinotecan as third-line therapy in patients with advanced colorectal cancer after failure to irinotecan, oxaliplatin and 5-fluorouracil Acta Oncologica. 2007; 46(5): 697-701. PMid: 17562448. https: //doi.org/10.1080/02841860601009455

[9] Schønnemann KR, Yilmaz M, Bjerregaard JK, et al. Phase II study of biweekly cetuximab in combination with irinotecan as secondline treatment in patients with platinum-resistant gastro-oesophageal cancer. European Journal of Cancer. 2012; 48(4): 510-517. PMid: 22244801. https://doi.org/10.1016/j.ejca.2011.12.005

[10] Pfeiffer P, Sorbye H, Qvortrup C, et al. Maintenance Therapy With Cetuximab Every Second Week in the First-Line Treatment of Metastatic Colorectal Cancer: The NORDIC-7.5 Study by the Nordic Colorectal Cancer Biomodulation Group. Clinical Colorectal Cancer. 2015; 14(3): 170-176. PMid: 25956187. https ://doi .org/10.1 016/j.clcc.2015.03.002

[11] Antonovsky A. Helbredets mysterium. Kbenhavn: Hans Reitzels Forlag; 2000. 1-231 p.

[12] Lindstrom B, Eriksson M. Salutogenesis. J Epidemiol Community Health. 2005; 59(6): 440-2. PMid: 15911636. https://doi.org/ 10.1136/jech. 2005.034777

[13] NCI. Cancer Therapy Evaluation Program, Common Terminology Criteria for Adverse Events, Version 3.0. 2006.

[14] Ouwerkerk J, Boers-Doets C. Best practices in the management of toxicities related to anti-EGFR agents for metastatic colorectal cancer. Eur J Oncol Nurs. 2010; 14(4): 337-49. PMid: 20580896 https://doi.org/10.1016/j.ejon.2010.03.004

[15] Baker N, Armour K, Meystre C, et al. PA14 The legacy of cancer: why a health promoting approach is so important in palliative care. 2015; 5(Suppl 1): A23-4. 